\title{
Calidad de vida y aspectos sociales como determinantes de fragilidad
}

\author{
A. Maseda, C. de Labra, L. Lorenzo-López, N. Cibeira, L. Regueiro-Folgueira, J.C. \\ Millán-Calenti
}

Universidade da Coruña, Grupo de Investigación en Gerontología, Instituto de Investigación Biomédica de A Coruña (INIBIC), Complexo Hospitalario Universitario de A Coruña (CHUAC), SERGAS, A Coruña, España

Objetivos: La disminución del apoyo social, el vivir solo o una baja calidad de vida son aspectos que se han identificado como factores de riesgo para presentar discapacidad, morbilidad o mortalidad, no habiéndose demostrado hasta el momento su influencia sobre la fragilidad. En este estudio pretendemos analizar la relación de los aspectos sociales con el fenotipo de fragilidad (prefragilidad y fragilidad).

Método: Se ha realizado estudio transversal con una muestra representativa $(n=749)$ de personas de 65 o más años de edad en el ámbito comunitario. Se recogieron datos sociodemográficos y se evaluaron los recursos sociales (mediante la escala OARS) y la calidad de vida (mediante la escala de la Organización Mundial de la Salud, WHOQOL-BREF). La fragilidad se definió utilizando los 5 criterios físicos de Fried (pérdida involuntaria de peso, fatiga, actividad física reducida, reducción de la velocidad de la marcha y reducción de la fuerza muscular), considerando la presencia de fragilidad si se cumplían al menos tres de los cinco criterios y prefragilidad si se cumplían uno o dos de ellos.

Resultados: El síndrome de fragilidad se ha relacionado principalmente con el género femenino, edad superior a 75 años y con mala calidad de vida general y mala calidad de vida enmarcada en el dominio de salud física del WHOQOL-BREF. Edad entre 80 y 89 años y baja calidad de vida en general fueron los principales determinantes de la presencia de fragilidad en los hombres. Por el contrario, en las mujeres la presencia de fragilidad solamente se vinculó al dominio de salud física del WHOQOL-BREF.

Conclusiones: La aparición de la fragilidad no solo depende de aspectos físicos, sino también de ciertos aspectos sociales o sociodemográficos y de la calidad de vida percibida por la persona mayor. Es importante incluir protocolos dirigidos a mejorar la calidad de vida, como factor de riesgo modificable, a fin de prevenir o revertir la aparición de la fragilidad en las personas mayores.

Este trabajo ha sido financiado por la Xunta de Galicia (ED431C2017/49 y Red FrailNet IN607C 2016/08). 